\title{
Análise da Segmentação e Extração de Características na Detecção de COVID-19 em imagens de Raio-x de Tórax
}

\author{
Camila Catiely de Sá Almondes ${ }^{1}$, Flávio Henrique Duarte de Araújo ${ }^{12}$ \\ ${ }^{1}$ Programa de Pós Graduação em Engenharia Elétrica, \\ Universidade Federal do Piauí - Teresina, Brasil \\ ${ }^{2}$ Departamento de Sistemas de Informação, \\ Universidade Federal do Piauí - Picos, Brasil \\ cctiely97@hotmail.com, flavio86@ufpi.edu.br
}

\begin{abstract}
COVID-19 mainly affects the lungs, causing shortness of breath, coughing and even multiple organ failure, making people seriously ill. A chest $X$-ray is necessary for testing and to assess the lung and the progress of the virus in terms of its effects. This work presents the evaluation of the descriptors Dense Net201, VGG16, RESNET50 and Xception, and the classifiers Multi-layer Perceptron (MLP) and Random Forest (RF), using the COVID-19 chest $x$-ray database for COVID-19 diagnosis. To evaluate the segmentation, the base Tuberculosis (TB) Chest X-ray Database was used. The tests were performed on a set of segmented images containing 6012 images of pulmonary infections, 3616 of COVID-19 and 10192 without findings. The evaluated scenarios were (Covid $x$ Normal), (Covid $x$ Lung Opacity), (Covid $x$ Lung Opacity $x$ Normal). The best results were achieved with the descriptor DenseNet201 and the MLP classifier in the scenario (Covid x Normal), with Accuracy and Kappa of 0.99.
\end{abstract}

Resumo. O COVID-19 afeta principalmente os pulmões, causando falta de ar, tosse e até falência de múltiplos órgãos, deixando as pessoas gravemente doentes. A radiografia de tórax se torna necessária para testes e para avaliar o pulmão e o progresso do vírus quanto aos seus efeitos. Este trabalho apresenta a avaliação dos descritores Dense Net201, VGG16, RESNET50 e Xception, e os classificadores Multi-layer Perceptron (MLP) e Random Forest (RF), com a utilização da base COVID-19 chest $x$-ray database para o diagnóstico do COVID-19. Para avaliar a segmentação foi utilizada a base Tuberculosis (TB) Chest X-ray Database. Os testes foram realizados em um conjunto de imagens segmentadas contendo 6012 imagens de infecções pulmonares, 3616 de COVID-19 e 10192 sem achados. Os cenários avaliados foram (Covid $x$ Normal), (Covid $x$ Opacidade Pulmonar), (Covid $x$ Opacidade Pulmonar $x$ Normal). Os melhores resultados foram alcançados com o descritor DenseNet201 e o classificador MLP no cenário (Covid x Normal), com Acurácia e Kappa de 0,99 .

\section{Introdução}

No ano de 2019, surgiu uma nova doença chamada Coronavírus (COVID-19). O primeiro caso apareceu em Wuhan, na China. A doença é extremamente contagiosa e se espalha rapidamente pelo mundo, apresentando sintomas comuns como febre, tosse, 
mialgia ou fadiga, causando um número crescente de mortes e de recepções em unidades de terapia intensiva (UTI). Portanto, é importante identificar casos positivos de COVID19 no estágio inicial, pois ajudará a isolar os pacientes o mais rápido possível e a quebrar a cadeia de transição [Afshar et al. 2020].

Sendo assim, a tomografia computadorizada (TC) e as radiografias de tórax são métodos utilizados para o diagnóstico por meio de ferramentas automatizadas para analisar imagens de TC e raios-X com base em inteligência artificial (IA). Dentre os métodos utilizados para análise, a escolha da radiografia é mais vantajosa que a tomografia, por ser um procedimento mais rápido, fácil, barato e menos prejudicial, pois este exame pode revelar pneumonia padrão proeminente de COVID-19, pois de acordo com a Organização Mundial da Saúde (OMS), a COVID-19 abre buracos nos pulmões como a síndrome respiratória aguda grave (SARS-VOC), fazendo-os parecer "favos de mel"'Narin et al. 2020]. Deste modo, é necessário desenvolver soluções para a análise de imagens de raios-X de tórax para investigações prematuras [Narin et al. 2020].

Os trabalhos [Tang et al. 2018], [Teixeira et al. 2020] demonstraram o impacto da segmentação do pulmão na identificação COVID-19 usando imagens de raio-x na rede U-net, já [Tang et al. 2018] utilizou uma R-CNN 3d inspirada na U-net e um classificador 3D. [Teixeira et al. 2020] também buscou avaliar quais características mais contribuem decisivamente para a identificação da patologia. $O$ trabalho utilizou as mesmas classes de imagens aplicadas neste artigo, sendo elas: Opacidade Pulmonar (Sem Covid), COVID19 e Normal, na classificação foram usadas três arquiteturas de redes neurais convolucionais (CNN): VGG, ResNet e Inception.

Para a descrição das imagens, comumente são utilizadas técnicas baseadas em CNNs, como no trabalho [da Nobrega et al. 2018], os autores utilizaram as seguintes arquiteturas para a extração de características das imagens: VGG16, VGG19, Xception, RESNET50, DenseNet169 e DenseNet201. Em seguida, as imagens foram classificadas utilizando os classificadores Naive bayes, MLP, SVM, K-NearNeighbors (KNN) e RF, alcançando Área Sob a Curva ROC (AUC) de 93,19\%. Na aplicação de descritores de textura e redes neurais, os autores [Varela-Santos and Melin 2021] apresentaram uma experimentação utilizando a GLCM, LBP e CNNs nas imagens com COVID-19. Os resultados obtidos alcançaram $94,30 \%$ de precisão.

Com base no exposto, o presente trabalho avalia os classificadores e descritores mais adequados para auxiliar especialistas na detecção e classificação de pacientes infectados com COVID-19 através das imagens de raio-X. Para o desenvolvimento dessa metodologia realizamos testes com os descritores DenseNet201, VGG16, RESNET50 e Xception, e os classificadores Multi-layer Perceptron (MLP) e Random Forest (RF). A principal contribuição do trabalho é avaliar se a segmentação melhora os resultados da classificação para a detecção de lesões pulmonares na região do tórax, bem como os classificadores e descritores mais adequados para o atual problema no diagnóstico do COVID-19.

\section{Materiais e Métodos}

Nesta seção, descrevemos a metodologia utilizada para segmentar as imagens e classificar as lesões pulmonares. Os estágios envolvidos nestas etapas são apresentados na Figura 1. A metodologia proposta está dividida em cinco etapas: A primeira consiste na 
aquisição das imagens da base COVID-19 Chest X-ray Database disponível no Kaggle. A segunda foca na segmentação da região do pulmão realizada pela a U-net, primeira etapa de treinamento realizada no repositório Tuberculosis (TB) Chest $X$-ray Database e suas respectivas máscaras, e no segundo experimento nas classes sem máscara. Na terceira etapa, foi realizado o pré-processamento das imagens utilizando a normalização 8-bits e a aplicação do CLAHE, seguindo para a quarta etapa, onde foi realizado a extração de características das lesões pulmonares utilizando os descritores: DenseNet201, VGG16, RESNET50 e Xception. Por fim, na etapa de classificação, foram avaliados os classificadores MLP e RF.

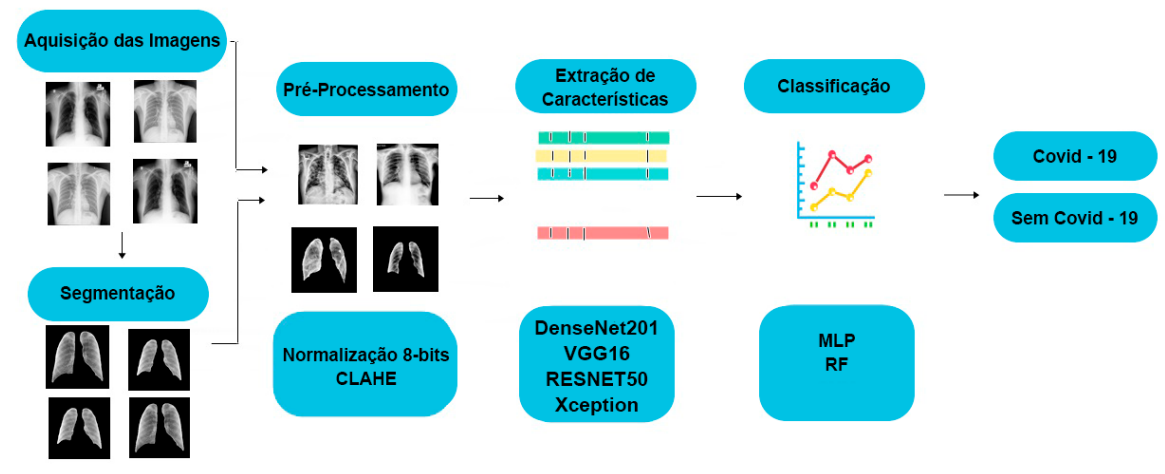

Figura 1. Fluxograma do método proposto dividido em cinco etapas.

\subsection{Aquisição das imagens}

Neste trabalho foram utilizados 2 repositórios públicos de imagens de raio-x do tórax. A primeira base é a Tuberculosis (TB) Chest X-ray Database [Rahman et al. 2020] que contém um conjunto de dados de máscaras pulmonares de Tuberculose correspondentes, que foram usadas apenas para treinar e avaliar o modelo de segmentação pulmonar. A base conta com 360 imagens de raios-X normais e 344 anormais (pulmão infectado somente com Tuberculose), totalizando 704 imagens de raio-X junto de suas respectivas máscaras pulmonares, todas anotadas por radiologistas especialistas. Portanto, a rede U-Net foi treinada e analisada com imagens normais e anormais.

O segundo repositório é o COVID-19 Chest X-ray Database [T. R. Muhammad 2020] que contém o conjunto de classes testadas no trabalho apenas para avaliar a classificação das imagens, na qual está sendo construída por uma equipe de pesquisadores de Doha e Qatar University no Catar e da University of Dhaka em Bangladesh, com seus colaboradores do Paquistão e da Malásia e através da colaboração com médicos, criaram um banco de dados contendo imagens de raios-X de tórax. O banco possui imagens com casos de: COVID-19 positivos, casos normais e de infecções pulmonares. Este repositório é atualizado gradualmente assim que surgem novos exames, o banco contém 3616 casos de COVID-19, juntamente com 10.192 imagens Normais, 6012 de Opacidade Pulmonar (Infecção Pulmonar Sem COVID-19).

\subsection{Pré-Processamento}

$\mathrm{Na}$ literatura, existem diversas bases de imagens disponíveis em repositórios públicos, mas por conta dos equipamentos de raio-x, possuem vários tamanhos e ex- 
tensões e isto faz com que apresentem algumas imagens de baixa qualidade. Para melhorar e tornar mais preciso os resultados no processo de detecção e classificação das lesões pulmonares, aplicou-se 2 técnicas de pré-processamento: a normalização 8-bits e Contrast limited adaptive histogram equalization (CLAHE).

Os conjunto de dados disponibilizados de COVID-19, variam de tipo entre 8, 16 e 32 bits. No entanto, a maioria das amostras são do tipo inteiro de 8 bits. Assim, todas as imagens foram convertidas para 8 bits. Para realçar o contraste, o CLAHE foi aplicado, seu funcionamento é basicamente dividir cada imagens em blocos, em vez da amostra inteira para equalizar os histogramas dessas regiões, regulando a amplificação do ruídos para assim obter resultados significativos. No pré-processamento foi definido a dimensão dos blocos em $8 \times 8$. A escolha de áreas menores foi para reduzir a probabilidade de propagação de ruídos. Porém, quando o ruído é encontrado, é aplicado o limite de corte com valor padrão igual a 2. Assim, se em qualquer divisão do histograma forem encontrados ruídos superiores ao valor de corte, os mesmos serão removidos e distribuídos antes da equalização do histograma.

\subsection{Segmentação}

Para a segmentação, foram utilizadas 704 imagens e suas respectivas máscaras da base de Tuberculose, que serviram para treinar a rede e segmentar a região do pulmão. Após obter os resultados através do conjunto de treino da base, utilizou-se respectivamente as classes: COVID-19, Opacidade Pulmonar e Normal para o conjunto de testes.

A rede U-net consiste em um caminho de contração e um caminho de expansão. O caminho de contração consiste na repetição de duas convoluções $3 \times 3$ (convoluções não preenchidas), cada uma possui um ReLU e um pooling máximo $2 \times 2$ e passo 2 para redução da dimensionalidade. Em cada etapa de redução, dobra-se o número de filtros dos canais. Cada etapa no caminho expansivo consiste em uma ampliação da amostragem do mapa de características seguido por uma convolução 2x2 ("up-convolution") que divide a metade do número de canais de características, uma concatenação com o correspondente recortado do mapa de características do caminho de contratação e duas convoluções 3 x 3, cada uma seguida por um ReLU. O corte é necessário devido à perda de pixels de borda em cada convolução. Na camada final, uma convolução 1 x 1 é usada para mapear 64 vetores de características de cada componente para o número desejado de classes. No total, a rede tem 23 camadas convolucionais.

Após a aplicação da U-net foi utilizado morfologia matemática para remover pequenas regiões de ruídos, refinar as porções segmentadas e definir os contornos apropriados para o método de segmentação. As operações com os respectivos parâmetros foram: erosão (disco de raio 2), dilatação em uma janela 2 x 2 e fechamento (disco de raio 10). A Figura 2 ilustra um exemplo do uso do CHAHE seguido pela segmentação.

\subsection{Extração de características e Classificação}

Após gerar as máscaras de segmentação da região do pulmão, as mesmas foram utilizadas para extrair apenas as características dessas regiões. As arquiteturas utilizadas foram pré-treinadas na ImageNet para a extração de características e os resultados foram coletados após a sequência de convoluções e pooling. Os descritores utilizados foram: DenseNet201 [Huang et al. 2017], VGG16 [Simonyan and Zisserman 2014], 


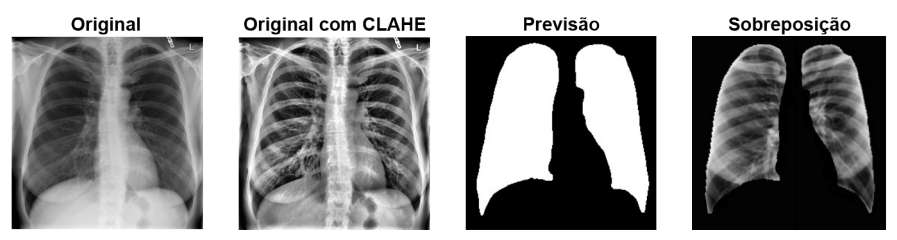

Figura 2. Segmentação do Pulmão no conjunto de imagens de COVID-19 Chest $X$-ray Database e aplicação do CLAHE.

RESNET50 [He et al. 2016] e Xception [Chollet 2017] com os seguintes argumentos: (weights ='imagenet', include_top $=$ False, o formato de entrada para todas as imagens foram input_shape $=(256,256,3)$, camada de pooling $=$ 'avg'). Já os classificadores utilizados foram: MLP [Nicolas 2015] com os seguintes critérios: (max_iter $=300$, alpha $=0.0001$, hidden_layer_sizes $=(200)$ e activation $=$ 'relu') e RF com (max_depth $=$ 50, n_estimators $=250$ ) [Suthaharan 2016].

\section{Resultados e discussão}

No modelo U-Net, foi utilizado o otimizador Adam, taxa de aprendizado 0.0005, mini-batch size de 16 com 30 épocas. A divisão do conjunto de dados das imagens de Tuberculose foi de $80 \%$ para treino (563 imagens) e 20\% teste (142 imagens), onde as imagens foram redimensionadas para 256x256. A segmentação na U-net obteve uma acurácia de 0,98 , Precisão de 0,97 e o Dice de 0,91 . O resultado demonstrou que a rede conseguiu delimitar adequadamente a região do pulmão, como pode ser visto na Figura 2.

A Figura 3 mostra que a rede não sofreu overfiting, pois o Dice e o erro obtidos tanto no conjunto de treino, como na validação, foram próximos durante o treinamento.
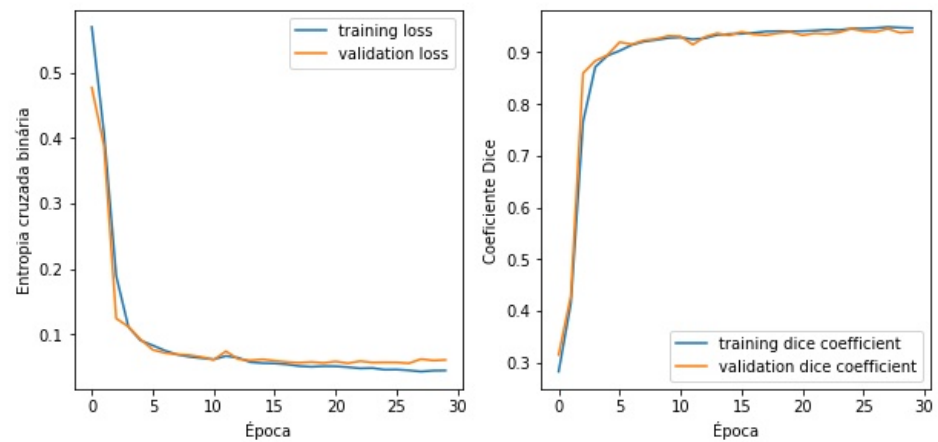

Figura 3. Decaimento do erro (direita) e b) Coeficiente Dice (esquerda).

As Tabelas 1 e 2 apresentam os melhores resultados e desempenho comparativo de cada descritor para as imagens (Sem Segmentação e Com Segmentação) que foram divididas em $70 \%$ para treino e $30 \%$ para teste, para a classificação de casos de COVID19 ou com lesões pulmonares (Sem COVID-19). Para isso, foram utilizadas as seguintes métricas: Acurácia (Ac), Kappa (Ka), Precisão (Pr) e Área Sob a Curva (AUC) - ROC.

$\mathrm{Na}$ Tabela $1 \mathrm{com}$ as redes treinadas com imagens de raios-X sem segmentação, o MLP obteve o melhor desempenho na classificação das imagens com os descritores 
Tabela 1. Melhores resultados de cada descritor (Sem Segmentação)

\begin{tabular}{|c|c|c|c|c|c|c|}
\hline Descritores & Classes & Classificador & Acurácia & Kappa & Precisão & AUC \\
\hline \multirow{6}{*}{ DenseNet201 } & \multirow{2}{*}{ Covid x Normal } & MLP & 0.99 & 0.99 & 0.99 & 0.99 \\
\hline & & RF & 0.98 & 0.95 & 0.97 & 0.98 \\
\hline & \multirow{2}{*}{ Covid x Opacidade Pulmonar } & MLP & 0.97 & 0.95 & 0.97 & 0.97 \\
\hline & & RF & 0.92 & 0.83 & 0.91 & 0.92 \\
\hline & \multirow{2}{*}{ Covid x Normal x Opacidade Pulmonar } & MLP & 0.98 & 0.97 & 0.97 & 0.98 \\
\hline & & $\mathrm{RF}$ & 0.94 & 0.90 & 0.91 & 0.95 \\
\hline \multirow{6}{*}{ RESNET50 } & \multirow{2}{*}{ Covid x Normal } & MLP & 0.99 & 0.99 & 0.99 & 0.99 \\
\hline & & RF & 0.95 & 0.88 & 0.93 & 0.95 \\
\hline & \multirow{2}{*}{ Covid x Opacidade Pulmonar } & MLP & 0.96 & 0.93 & 0.96 & 0.96 \\
\hline & & RF & 0.93 & 0.85 & 0.92 & 0.92 \\
\hline & \multirow{2}{*}{ Covid x Normal x Opacidade Pulmonar } & MLP & 0.97 & 0.96 & 0.97 & 0.98 \\
\hline & & RF & 0.90 & 0.83 & 0.87 & 0.92 \\
\hline \multirow{6}{*}{ VGG16 } & \multirow{2}{*}{ Covid x Normal } & MLP & 0.95 & 0.90 & 0.95 & 0.95 \\
\hline & & RF & 0.90 & 0.80 & 0.89 & 0.90 \\
\hline & \multirow{2}{*}{ Covid x Opacidade Pulmonar } & MLP & 0.95 & 0.90 & $\mathbf{0 . 9 5}$ & 0.95 \\
\hline & & RF & 0.90 & 0.79 & 0.89 & 0.90 \\
\hline & \multirow{2}{*}{ Covid x Normal x Opacidade Pulmonar } & MLP & 0.97 & 0.96 & 0.96 & 0.98 \\
\hline & & RF & 0.92 & 0.87 & 0.88 & 0.93 \\
\hline \multirow{6}{*}{ Xception } & \multirow{2}{*}{ Covid x Normal } & MLP & 0.99 & 0.99 & 0.99 & 0.99 \\
\hline & & RF & 0.98 & 0.96 & 0.97 & 0.98 \\
\hline & \multirow{2}{*}{ Covid x Opacidade Pulmonar } & MLP & 0.91 & $\mathbf{0 . 8 1}$ & $\mathbf{0 . 9 0}$ & 0.91 \\
\hline & & RF & 0.84 & 0.65 & 0.82 & 0.83 \\
\hline & \multirow{2}{*}{ Covid x Normal x Opacidade Pulmonar } & MLP & 0.95 & 0.92 & 0.93 & 0.96 \\
\hline & & $\mathrm{RF}$ & 0.90 & 0.83 & 0.84 & 0.91 \\
\hline
\end{tabular}

RESNET50, Xception e DenseNet201 no cenário Covid x Normal apresentando Acurácia, Kappa, Precisão e AUC de 0,99. A MLP conseguiu os melhores resultados, porque esse classificador se adapta melhor para grande quantidade de características e numéricas, que são as extraídas pelas redes pré-treinadas. Na categoria Covid x Opacidade Pulmonar, o MLP em conjunto com a DenseNet201 obteve resultados superiores aos outros descritores com Acurácia, Precisão e AUC de 0,97. A DenseNet201 é uma rede mais profunda que as outras arquiteturas, além disso, na junção de mapas de caraterísticas de diferentes camadas, a DenseNet201 aumenta a variedade de características nas entradas e melhora a eficiência, sendo uma das principais diferenças apresentadas em relação as outras redes, e assim obtendo melhores resultados também na classificação multiclasses entre Covid x Normal x Opacidade Pulmonar com Acurácia e AUC de 0,98.

Os resultados apresentados na Tabela 2 mostram que o melhor desempenho obtido foi do classificador MLP juntamente com os descritor RESNET50 alcançando uma Acurácia de 0,93 nas classes Covid x Normal, no cenário Covid x Opacidade Pulmonar apresentaram a Precisão de 0,90 e na categoria Covid x Normal x Opacidade Pulmonar com 0,90 de AUC nas imagens com segmentação. A rede pré-treinada RESNET50 é conhecida por ser relativamente fácil de ser otimizada e pode atingir maior precisão, como é um descritor de rede neural de camadas profundas, sendo assim obteve bons resultados nas imagens com segmentação. Foram extraídas 2048 características na última camada de pooling da RESNET50.

O modelo U-net treinado no conjunto de dados de raios-X de tórax de Tuberculose do Kaggle pode segmentar as áreas pulmonares das imagens de raios-X do banco de dados de classificação de forma confiável, de acordo com as métricas apresentadas na Tabela 2. Por outro lado, as imagens de raios-X de tórax de pulmão segmentado forneceram uma visão de diminuição de desempenho para todas as redes testadas em comparação com resultados nas imagens sem segmentação, por isto vale ressaltar que utilizar as imagens sem segmentação é preferível neste caso. Isso reflete o fato de que, rasas ou profundas, todas as CNNs não conseguiram distinguir COVID-19 de pulmões normais ou infecções pulmo- 
Tabela 2. Melhores resultados de cada descritor (Com Segmentação)

\begin{tabular}{|c|c|c|c|c|c|c|}
\hline Descritores & Classes & Classificador & Acurácia & Kappa & Precisão & AUC \\
\hline \multirow{6}{*}{ DenseNet201 } & \multirow{2}{*}{ Covid x Normal } & MLP & 0.93 & 0.83 & 0.90 & 0.92 \\
\hline & & RF & 0.87 & 0.63 & 0.79 & 0.86 \\
\hline & \multirow{2}{*}{ Covid x Opacidade Pulmonar } & MLP & 0.90 & 0.80 & 0.90 & 0.90 \\
\hline & & RF & 0.83 & 0.64 & 0.81 & 0.85 \\
\hline & \multirow{2}{*}{ Covid x Normal x Opacidade Pulmonar } & MLP & 0.87 & 0.79 & 0.85 & 0.89 \\
\hline & & $\mathrm{RF}$ & 0.89 & 0.65 & 0.72 & 0.84 \\
\hline \multirow{6}{*}{ RESNET50 } & \multirow{2}{*}{ Covid x Normal } & MLP & 0.93 & $\mathbf{0 . 8 3}$ & $\overline{0.91}$ & 0.92 \\
\hline & & $\mathrm{RF}$ & 0.88 & 0.67 & 0.81 & 0.88 \\
\hline & \multirow{2}{*}{ Covid x Opacidade Pulmonar } & MLP & 0.91 & 0.81 & 0.90 & 0.90 \\
\hline & & RF & 0.84 & 0.66 & 0.82 & 0.84 \\
\hline & \multirow{2}{*}{ Covid x Normal x Opacidade Pulmonar } & MLP & 0.88 & 0.81 & 0.86 & 0.90 \\
\hline & & RF & 0.81 & 0.68 & 0.73 & 0.86 \\
\hline \multirow{6}{*}{ VGG16 } & \multirow{2}{*}{ Covid x Normal } & MLP & 0.92 & 0.81 & $\mathbf{0 . 8 9}$ & 0.91 \\
\hline & & RF & 0.88 & 0.67 & 0.80 & 0.88 \\
\hline & \multirow{2}{*}{ Covid x Opacidade Pulmonar } & MLP & $\mathbf{0 . 8 8}$ & $\mathbf{0 . 7 5}$ & $\mathbf{0 . 8 8}$ & 0.87 \\
\hline & & RF & 0.82 & 0.62 & 0.80 & 0.82 \\
\hline & \multirow{2}{*}{ Covid x Normal x Opacidade Pulmonar } & MLP & 0.87 & 0.78 & 0.84 & 0.89 \\
\hline & & RF & 0.81 & 0.67 & 0.72 & 0.86 \\
\hline \multirow{6}{*}{ Xception } & \multirow{2}{*}{ Covid x Normal } & MLP & 0.92 & 0.80 & 0.89 & 0.90 \\
\hline & & RF & 0.89 & 0.69 & 0.82 & 0.88 \\
\hline & \multirow{2}{*}{ Covid x Opacidade Pulmonar } & MLP & $\mathbf{0 . 8 7}$ & 0.72 & 0.86 & 0.86 \\
\hline & & RF & 0.80 & 0.56 & 0.77 & 0.79 \\
\hline & \multirow{2}{*}{ Covid x Normal x Opacidade Pulmonar } & MLP & 0.85 & 0.76 & 0.82 & 0.88 \\
\hline & & $\mathrm{RF}$ & 0.79 & 0.64 & 0.70 & 0.83 \\
\hline
\end{tabular}

nares com uma confiabilidade alta quando apenas as regiões pulmonares são usadas como entrada para as CNNs. Este aspecto mostra que mesmo que a lesão da COVID-19 afete apenas região do pulmão, os algoritmos estão levando em consideração características de outras regiões do tórax para a tomada de decisão. Os testes se tornam necessários para criar e simular cenários verdadeiros e complexos onde as doenças com lesões pulmonares compartilham muitas características, tornando imprescindível uma propagação adequada por parte dos modelos.

Em resumo, o MLP teve um bom desempenho nos pulmões segmentados e sem segmentação, o que reflete que a rede mais profunda pode classificar com mais precisão para imagens de raios-X de pulmão. No entanto, como o estudo de caso é um problema binário e de multiclasses semelhantes, as regiões dos pulmões das imagens de COVID-19 não são significativamente diferentes das imagens de patologias similares, então todos os teste conseguiram um bom desempenho na generalização das categorias.

\section{Conclusão}

Neste trabalho, foi realizado uma avaliação dos classificadores e descritores neurais mais adequados para auxiliar especialistas na detecção e classificação do COVID-19. Os melhores resultados da metodologia foram alcançados com o classificador MLP e o descritor DenseNet201 para as imagens sem segmentação. Os resultados obtidos utilizando a segmentação dos pulmões na extração de características demonstraram que, mesmo com essa propriedade aplicada as imagens, não foi possível melhorar os resultados da classificação, pois durante a classificação das imagens o algoritmo também está levando em consideração informações externas a região demarcada do pulmão.

Pretende-se em trabalhos futuros, encontrar melhores parâmetros para cada classificador e descritor abordado neste trabalho, como também realizar testes utilizando outras redes pré-treinadas, de segmentação e a combinação de características de diferentes descritores. 


\section{Referências}

Afshar, P., Heidarian, S., Naderkhani, F., Oikonomou, A., Plataniotis, K. N., and Mohammadi, A. (2020). Covid-caps: A capsule network-based framework for identification of covid-19 cases from X-ray images. arXiv preprint arXiv:2004.02696.

Chollet, F. (2017). Xception: Deep learning with depthwise separable convolutions. In Proceedings of the IEEE conference on computer vision and pattern recognition, pages 1251-1258.

da Nobrega, R. V. M. et al. (2018). Lung nodule classification via deep transfer learning in ct lung images. In 2018 IEEE 31st International Symposium on Computer-Based Medical Systems (CBMS), pages 244-249.

He, K., Zhang, X., Ren, S., and Sun, J. (2016). Deep residual learning for image recognition. In Conference on Computer Vision and Pattern Recognition, volume 2016December, pages 770-778. IEEE Computer Society.

Huang, G., Liu, Z., Van Der Maaten, L., and Weinberger, K. Q. (2017). Densely connected convolutional networks. In Proceedings of the IEEE conference on computer vision and pattern recognition, pages 4700-4708.

Narin, A., Kaya, C., and Pamuk, Z. (2020). Automatic detection of coronavirus disease (covid-19) using x-ray images and deep convolutional neural networks. arXiv preprint arXiv:2003.10849.

Nicolas, P. R. (2015). Scala for machine learning. Packt Publishing Ltd.

Rahman, T., Khandakar, A., Kadir, M. A., Islam, K. R., Islam, K. F., Mazhar, R., Hamid, T., Islam, M. T., Kashem, S., Mahbub, Z. B., et al. (2020). Reliable tuberculosis detection using chest $\mathrm{x}$-ray with deep learning, segmentation and visualization. IEEE Access, 8:191586-191601.

Simonyan, K. and Zisserman, A. (2014). Very deep convolutional networks for large-scale image recognition. arXiv preprint arXiv:1409.1556.

Suthaharan, S. (2016). Machine learning models and algorithms for big data classification. Integr. Ser. Inf. Syst, 36:1-12.

T. R. Muhammad, E. H. Chowdhury, A. K. R. M. M. A. K. Z. B. M. e. a. (2020). Covid-19 chest x-ray database. online. Available: https://www.kaggle.com/tawsifurrahman/covid19-radiography-database.

Tang, H., Kim, D. R., and Xie, X. (2018). Automated pulmonary nodule detection using 3d deep convolutional neural networks. In 2018 IEEE 15th International Symposium on Biomedical Imaging (ISBI 2018), pages 523-526.

Teixeira, L. O., Pereira, R. M., Bertolini, D., Oliveira, L. S., Nanni, L., Cavalcanti, G. D., and Costa, Y. M. (2020). Impact of lung segmentation on the diagnosis and explanation of covid-19 in chest X-ray images. arXiv preprint arXiv:2009.09780.

Varela-Santos, S. and Melin, P. (2021). A new approach for classifying coronavirus covid-19 based on its manifestation on chest x-rays using texture features and neural networks. Information sciences, 545:403-414. 